\title{
New Tools For Correlative Light And Electron Microscopy
}

\author{
Matthias F. Langhorst ${ }^{1}$, Alevtyna Yakushevska ${ }^{2}$, Ben Lich $^{2}$ \\ ${ }^{1}$ TILL Photonics an FEI Company, Lochhamer Schlag 21, D-82166 Graefelfing, Germany \\ ${ }^{2}$ FEI, Achtseweg Noord 5, 5651 GG Eindhoven, The Netherlands
}

Combining information from light and electron microscopy adds significant value to biological imaging. The contextual information at the ultrastructural level as provided by electron microscopy perfectly complements the specificity, sensitivity and most of all live-cell-imaging capabilities of fluorescence microscopy. However, correlating information from both modalities still remains an experimental challenge. Due to the necessary sample preparation steps between (live cell) fluorescence imaging and electron microscopy, quick relocation to the area of interest, optimal contrast in both modalities and precise correlation between the two modalities is often hard to achieve.

FEI has recently introduced new solutions to overcome these experimental hurdles: CorrSight, a dedicated light microscopy system offering CLEM-specific functionality and automation of important workflow steps; MAPS, a software tool bridging the modalities to increase ease of use; and iCorr, a light microscope module integrated into the Tecnai family of transmission electron microscopes. These tools address different correlative workflows helping to optimize efficiency and data quality across the full range of CLEM experiments.

CorrSight uses an unsual geometry for a light microscope - while the stage is absolutely immobile, the whole microscopy system is moved for area scanning. This in combination with its self-contained, boxed design allows for innovative solutions to provide optimal sample support for different workflows in correlative light and electron microscopy. To automate time-resolved live-cell imaging with chemical fixation, a microfluidics device is integrated with an incubated sample area allowing for live cell imaging, event-triggered fixation and subsequent processing of the sample for electron microscopy. For different applications, a dedicated cryo stage allows contamination-free imaging of vitrified samples at high resolution. These special sample environments are complemented by a wide choice of light microscopy components ranging from a high-sensitivity widefield system to a spinning disc confocal with laser manipulation. The whole system aims at a very high degree of automation throughout the workflow, at the core of which is its control by MAPS, a powerful software package that also controls the EM acquisition so that cross-instrument tasks can be easily defined.

Apart from controlling CorrSight, MAPS also allows for correlation of light microscopy data captured on any light microscope with EM data acquisition on the full range of FEI SEMs / SDBs. To allow utmost flexibility in the choice of the light microscope, there is absolutely no dependence on any special hardware - correlation is carried out only on image data. Thus, existing or specialized light microscopy setups can be easily used for CLEM experiments and correlation can be carried out on any feature visible in both modalities.

FEI's integrated light and electron microscope (iCorr) combines a fluorescence light microscope and an electron microscope into a single harmonized instrument. This gives users a faster and 
more accurate approach to correlative microscopy. The iCorr is designed to greatly speed up and automate CLEM experiments, resulting in LM image stitches covering a large area of the sample, and automated LM-TEM image overlays at defined positions of interest.

The Tecnai iCorr consists of a fully integrated LED based widefield fluorescence microscope (FM) located at the normal sample position on the TEM column. Imaging in FM and TEM mode is done sequentially without manually exchanging the sample between the two imaging modes. Using the common EM specimen stage and specimen holder, the sample is tilted to $90^{\circ}$ in order to record LM images in reflection mode and fluorescence mode. The sample is then tilted to $0^{\circ}$ for normal TEM imaging. 